\title{
Análisis del portafolio como herramienta evaluativa de las prácticas clínicas de enfermería comunitaria en estudiantes de pregrado
}

\author{
P. Serrano-Gallardo a, M. Martínez-Marcosª, M.P. Arroyo-Gordo a , D. Lanza-Escobedo ${ }^{\text {b }}$
}

Objetivos. Analizar la implementación y la utilidad del portafolio como herramienta evaluativa de las prácticas clínicas en enfermería comunitaria en la Escuela de Enfermería Puerta de Hierro de la Universidad Autónoma de Madrid (UAM) y valorar la influencia de variables sociodemográficas y académicas en el proceso de evaluación continua del portafolio. Sujetos y métodos. Estudio descriptivo en el marco de un proyecto de innovación docente financiado por la UAM. La población objeto de estudio fueron estudiantes de segundo y tercer curso de enfermería de la escuela Puerta de Hierro (UAM), que realizaron sus prácticas clínicas de enfermería comunitaria en el medio de la atención primaria de salud $(n=38)$. Variables principales: calificaciones intermedias y finales en los trabajos que componían el portafolio (diario reflexivo, caso clínico, análisis de salud de la comunidad), puntualidad en las entregas, dificultad, utilidad y mejora del aprendizaje percibida, horas dedicadas a la semana, autoevaluación de las prácticas clínicas, calificación de la tutora, evaluación final del portafolio. Para el análisis bivariante se usaron pruebas no paramétricas. Se calcularon intervalos de confianza para un 95\% de seguridad. Resultados. Se analizaron 38 estudiantes con una edad media 21,5 \pm 4,3 años, $81,6 \%$ mujeres. Para el $56,7 \%$, la dificultad fue alta. La utilidad se señaló como alta en el $72,9 \%$ de los casos. La media en la autoevaluación del estudiante fue de 7,68; la evaluación de la tutora, de 8,31, y la calificación definitiva del portafolio final por parte de las profesoras, de 7,07. Conclusiones. La implementación del portafolio ha puesto de manifiesto la dificultad sentida por los estudiantes en un proceso que requiere escritura reflexiva, trabajo diario y autoevaluación. Ahora bien, estas dificultades se traducen en una alta percepción de utilidad y de mejora para el aprendizaje.

Palabras clave. Aprendizaje. Atención primaria. Educación enfermera de pregrado. Enfermería comunitaria. Espacio Europeo de Educación Superior. Portafolio. Prácticas clínicas.

\section{Portfolio analysis as evaluation tool of Community Nursing Clinical Practice Learning in undergraduates}

Aim. To analyze the implementation and usefulness of the portfolio as assessment tool for Community Nursing Clinical Practice Learning in the Puerta de Hierro Nursing School of Universidad Autónoma de Madrid (UAM), and to assess the influence of sociodemographic and academic variables in the process of continuous evaluation of the portfolio. Subjects and methods. cross-sectional study in the context of a teaching innovation project funded by the UAM. The target population: students of 2nd and 3rd courses of Puerta de Hierro Nursing School (UAM) that made their Community Nursing Clinical Practice Learning in the setting of the Primary Health Care $(n=38)$. Main outcomes: intermedium and final scores in the works that includes the portfolio ('reflective diary,' 'clinical case' and 'analysis of community health'), timeliness of delivery, difficulty, usefulness and perceived improvement of learning, hours spent/week, student selfassessment, qualification of the tutor, final evaluation of the portfolio. Bivariate analysis was done using nonparametric tests. Confidence intervals were calculated for $95 \%$ confidence. Results. 38 students were studied. The mean age was of $21.5 \pm 4.3$ years-old, $81.6 \%$ women. Difficulty was high in $56.7 \%$. The utility was noted as high in $72.9 \%$. The student self-assessment average score was 7.68, the evaluation of the tutor: 8.31 and portfolio final score by the teachers: 7,07. Conclusions. The implementation of portfolio has shown the difficulty felt by students in a process requiring reflective writing, daily work and self-evaluation. However, these difficulties translate into a high perception of usefulness and improve learning.

Key words. Clinical practice learning. Community nursing. European Higher Education Area. Learning. Nursing education. Portfolio. Primary care.
Escuela de Enfermería Puerta de Hierro. Universidad Autónoma de Madrid (UAM). b Departamento de Psicología Evolutiva y de la Educación. Facultad de Psicología. UAM. Majadahonda, Madrid, España.

Correspondencia Pilar Serrano-Gallardo. Escuela de Enfermería Puerta de Hierro. Joaquín Rodrigo, 2. E-28222

Majadahonda (Madrid).

E-mail pserranohpth@salud. madrid.org

Financiación Oficina para la Convergencia Europea de la Universidad Autónoma de Madrid (B6-07, n. 200700003837 ). 


\section{Introducción}

En la década de los años noventa se emprendió en Europa un proceso para la convergencia de la educación superior que, entre otras cuestiones relevantes, remarcaba la necesidad de innovar en los procesos de enseñanza-aprendizaje. Las universidades españolas inician entonces un camino de transformación para ajustarse a lo señalado en la Declaración de la Sorbona de 1998 y la Declaración de Bolonia, firmada el 19 de junio de 1999 por ministros con competencias en educación superior de 29 países europeos y que da su nombre al 'Proceso de Bolonia', proyecto de carácter intergubernamental con participación de universidades, estudiantes, la Comisión Europea y otras organizaciones, que tuvo como meta el establecimiento de un Espacio Europeo de Educación Superior y que culmina en el año 2010, plazo máximo otorgado para poner en marcha las nuevas enseñanzas de grado [1-3].

Las propuestas de reformas universitarias impulsadas por las nuevas directrices se han desarrollado en función del establecimiento de las competencias finales que los estudiantes deben conseguir en cada titulación y que constituyen el eje de todo proceso de enseñanza-aprendizaje, modificando sustancialmente la manera en la que el docente ha de planificar la enseñanza.

Algunas técnicas pedagógicas, entre las que se encuentra el portafolio, integran los parámetros de evaluación del aprendizaje y de desarrollo competencial, dado que es una herramienta de construcción de conocimientos que incorpora una dinámica de reflexión y autoevaluación. Se convierte de esta manera en una metodología de enseñanza y de evaluación [4].

Diferentes definiciones de 'portafolio' ponen de manifiesto los rasgos operativos de esta herramienta de evaluación:

- Logros que debe alcanzar el estudiante en su proceso de aprendizaje [5].

- Colección de trabajos del estudiante elaborados a partir de sus experiencias reales, que pueden ser acordados con el profesor y también seleccionados por el propio alumno [6].

- Ha de contener también una parte de escritura reflexiva del estudiante en torno a estos trabajos [7].

- Se han de producir encuentros sistemáticos entre las figuras implicadas en el proceso edu- cativo (profesor-estudiante, tutor-estudiante, profesor-tutor), con la finalidad de que se produzca feedback en el proceso evaluativo [8].

- Recoge los juicios de evaluación a partir de la observación y de los informes de un experto clínico y del tutor de prácticas clínicas [8].

Existes pocas experiencias con estas nuevas herramientas pedagógicas en nuestro entorno, especialmente en el ámbito de la formación de pregrado de enfermería [8-12], y sobre todo hay una carencia en el área de las prácticas clínicas.

En el curso académico 2006-2007, la Escuela de Enfermería Puerta de Hierro comenzó a desarrollar un proyecto de innovación docente, financiado por la Universidad Autónoma de Madrid (UAM) en el marco del Espacio Europeo de Educación Superior, centrado en la evaluación de las prácticas clínicas a través del portafolio en la materia de enfermería comunitaria.

Los objetivos del presente trabajo fueron analizar la implementación y la utilidad del portafolio como herramienta evaluativa de las prácticas clínicas en enfermería comunitaria en la Escuela de Enfermería Puerta de Hierro (UAM), y valorar la influencia de variables sociodemográficas $y$ académicas en el proceso de evaluación continua mediante el portafolio.

Los resultados de este estudio pueden contribuir a encontrar claves para mejorar el proceso de enseñanza-aprendizaje y, en última instancia, el desarrollo de competencias que necesitan los futuros profesionales enfermeros para brindar cuidados de calidad que favorezcan un buen nivel de salud de la población.

\section{Sujetos y métodos}

Se llevó a cabo un estudio observacional descriptivo transversal con componentes longitudinales para el análisis.

La población objeto de estudio fueron los estudiantes de segundo y tercer curso de Enfermería de la Escuela Puerta de Hierro (UAM), que realizaron su primer periodo de prácticas clínicas de enfermería comunitaria, en el medio de la atención primaria de salud, en tres áreas de salud de la Comunidad de Madrid, en el año académico 2007-2008 $(n=38)$. El periodo de prácticas tenía una duración de cinco semanas. Tanto a 
estudiantes como a tutores se les entregó la guía de prácticas clínicas de cada uno de los cursos y en la que se reflejaban, entre otras cuestiones, las competencias a desarrollar durante su estancia y los objetivos pedagógicos que les ayudarían para tal propósito. Las competencias generales comunes a ambos cursos eran:

- Brindar cuidados integrales, integrado y continuos a individuos y familias.

- Identificar las necesidades de cuidados enfermeros, establecer objetivos, ejecutar y evaluar los cuidados enfermeros a los grupos de pacientes incluidos en los diversos programas de salud.

- Aplicar en la práctica un método sistemático y organizado, que le permita definir problemas y seleccionar intervenciones.

- Ser capaz de tomar decisiones con autonomía.

- Comunicarse con los pacientes respetando sus valores, creencias y condiciones culturales, así como su condición intelectual y emocional.

- Mantener relaciones, comunicarse con los usuarios y familia en una atmósfera de confidencialidad y empatía que favorezca la comunicación.

- Trabajar en equipo, colaborar con otros sectores e instituciones, y asumir roles de liderazgo.

- Ser capaz de realizar intervenciones con la comunidad en diversos ámbitos de actuación: escuela, domicilio, asociaciones, centro de salud.

- Ser capaz de gestionar intervenciones de educación para la salud.

- Utilizar adecuadamente los soportes documentales que existan en el centro de salud.

- Realizar intervenciones enfermeras en los principales programas de salud, una competencia específica para segundo curso.

- Analizar la situación de salud de una comunidad, utilizando herramientas epidemiológicas, una competencia específica para tercer curso.

Para la elaboración del portafolio, además de la revisión bibliográfica [4-9,13-15], se realizó un envío por correo electrónico a todas las escuelas universitarias de enfermería españolas con la intención de recoger las experiencias que se estuvieran llevando a cabo con el portafolio como herramientas de evaluación. Esta recogida de información se completó con la visita programada a dos escuelas de universidades catalanas (Universitat de Manresa, adscrita a la Universitat
Autònoma de Barcelona, y Universitat Pompeu Fabra), las cuales estaban llevando a cabo experiencias muy aplicables al proyecto que se quería poner en marcha en nuestra escuela.

Todo el proceso descrito permitió el diseño del portafolio de prácticas clínicas, que siguió las siguientes etapas:

1. Elección del tipo de portafolio.

2. Construcción de la hoja de presentación del portafolio a los estudiantes.

3. Selección de los contenidos a incluir en el portafolio.

4. Forma de presentación del portafolio.

5. Propuesta de criterios de evaluación a estudiantes y tutores clínicos.

6. Definición de estrategias de comunicación.

La herramienta desarrollada fue un portafolio cerrado que comprendía los siguientes trabajos: diario reflexivo, caso clínico realizado sobre un paciente, un documento de análisis de la salud de la comunidad (ASC) y un documento de autoevaluación del estudiante. Como herramienta evaluativa adicional se incluía el informe de evaluación de la tutora de prácticas clínicas. Se pretendió que el portafolio contribuyera tanto a una evaluación sumativa (contenía trabajos que certificaban el nivel de competencia en el aprendizaje), formativa (incluía informes acerca del progreso de los estudiantes que posibilitaban la retroalimentación), como formadora (la metodología del portafolio permitía dar luz del proceso de aprendizaje con estrategias reflexivas y de autoevaluación) [14].

Respecto al proceso de implementación, cada alumno tenía asignado una tutora de prácticas en los centros de Atención Primaria y cuatro profesoras estaban encargadas de la supervisión y evaluación de las prácticas clínicas (10 estudiantes por cada profesora) a través del portafolio. A lo largo del periodo de prácticas clínicas, el estudiante debía realizar al menos tres entregas (vía correo electrónico) del diario, dos del caso y una de ASC. La profesora debía devolverle su evaluación en el plazo máximo de cuatro días (vía correo electrónico). Tanto para la evaluación de trabajos intermedios como para la del portafolio final, se crearon indicadores que de manera sistemática eran utilizados por las cuatro profesoras. Para la generación de dichos indicadores se partió de los propuestos por la Escuela 
Tabla I. Indicadores de evaluación de los trabajos que componían el portafolio.

\begin{tabular}{|c|c|c|c|}
\hline \multirow{3}{*}{ Trabajo } & \multirow{3}{*}{ Indicadores } & \multicolumn{2}{|c|}{ Ejemplo de operacionalización de un indicado } \\
\hline & & Nombre & Categorías ordinales \\
\hline & & & 1 \\
\hline $\begin{array}{l}\text { Diario } \\
\text { reflexivo }\end{array}$ & $\begin{array}{l}\text { A. Ortografía } \\
\text { B. Sintaxis } \\
\text { C. Utilización de un lenguaje adecuado } \\
\text { D. Nivel de reflexión sobre lo que sabe hacer } \\
\text { y aquello que se ha trabajado a la escuela } \\
\text { E. Nivel de reflexión sobre lo que aprende } \\
\text { y aquello que tendría que aprender } \\
\text { F. Nivel de reflexión sobre las relaciones interpersonales } \\
\text { G. Evidencias sobre la capacidad para la resolución } \\
\text { de problemas } \\
\text { H. El alumno realiza los cambios y mejoras } \\
\text { que propone la profesora }\end{array}$ & $\begin{array}{l}\text { D. Nivel de reflexión } \\
\text { sobre lo que } \\
\text { sabe hacer y } \\
\text { aquello que se } \\
\text { ha trabajado } \\
\text { a la escuela }\end{array}$ & $\begin{array}{l}\text { Las referencias a } \\
\text { los conocimientos } \\
\text { del estudiante de } \\
\text { aplicación directa a las } \\
\text { prácticas clínicas son } \\
\text { inexistentes a lo largo } \\
\text { de todo el diario }\end{array}$ \\
\hline Caso clínico & $\begin{array}{l}\text { A. Ortografía } \\
\text { B. Sintaxis } \\
\text { C. Utilización de un lenguaje adecuado } \\
\text { D. Valoración y diagnóstico del caso } \\
\text { E. Evolución del paciente } \\
\text { F. El alumno realiza los cambios y mejoras } \\
\quad \text { que propone la profesora }\end{array}$ & $\begin{array}{l}\text { D. Valoración y } \\
\text { diagnóstico } \\
\text { del caso }\end{array}$ & $\begin{array}{l}\text { Los datos son } \\
\text { insuficientes y se } \\
\text { han indagado de } \\
\text { manera no rigurosa } \\
\text { a lo largo de toda la } \\
\text { valoración. Existe una } \\
\text { desconexión absoluta } \\
\text { entre la valoración y } \\
\text { los diagnósticos del } \\
\text { paciente/usuario }\end{array}$ \\
\hline $\begin{array}{l}\text { Análisis de } \\
\text { comunidad }\end{array}$ & $\begin{array}{l}\text { A. Ortografía } \\
\text { B. Sintaxis } \\
\text { C. Utilización de un lenguaje adecuado } \\
\text { D. Descripción y análisis de la zona básica } \\
\text { de salud donde se ubica el centro } \\
\text { y el equipo de atención primaria } \\
\text { E. Describir la estructura física y funcional del } \\
\text { centro de salud, así como los roles del equipo } \\
\text { multidisciplinario ( } 2 .^{\circ} \text { curso). Descripción } \\
\text { y análisis del trabajo que realiza el equipo } \\
\text { enfermero ( } 3 .^{\text {er }} \text { curso) } \\
\text { F. El alumno realiza los cambios y mejoras } \\
\text { que propone la profesora }\end{array}$ & $\begin{array}{l}\text { D. Descripción y } \\
\text { análisis de la zona } \\
\text { básica de salud } \\
\text { donde se ubica el } \\
\text { centro y el equipo } \\
\text { de atención } \\
\text { primaria }\end{array}$ & $\begin{array}{l}\text { El documento no } \\
\text { describe ni adecuada } \\
\text { ni suficientemente } \\
\text { la situación de } \\
\text { salud (indicadores } \\
\text { sociodemográficos } \\
\text { y sanitarios) de la } \\
\text { población de la zona }\end{array}$ \\
\hline
\end{tabular}

de Ciencias de la Salud (Fisioterapia) de Manresa (Universitat Autònoma de Barcelona-Fundació Universitària del Bages), los cuales se adaptaron al proyecto de la Escuela Puerta de Hierro y se consensuaron entre las cuatro profesoras mencionadas. Dichos indicadores se operacionalizaron inicialmente mediante una escala ordinal de cinco categorías (1, incompleto, a 5, excelente)
(Tabla I); la puntuación obtenida en cada trabajo era la suma del valor obtenido de cada indicador. Posteriormente se recodificaron dichas puntuaciones totales a una escala de calificación estándar de 0 a 10. También se planificó un encuentro grupal estudiantes-profesor al inicio de las prácticas clínicas y uno individual tras finalizar dichas prácticas, una vez que ya había entrega- 
(1, incompleto, a 5, excelente)

2

Las referencias a los conocimientos del estudiante y a aquellos que puede aplicar a las prácticas clínicas a lo largo del diario son irrelevantes y sin ningún tipo de análisis crítico

3

Hay referencias a los conocimientos del estudiante y a aquellos que puede aplicar, pero no son constantes
Las referencias a los conocimientos del estudiante son constantes a lo largo de todo el diario y muestran un alto nivel de reflexión y análisis
Las referencias de la aplicación de los conocimientos logrados en la escuela son constantes, con un alto grado de análisis, y está claro determinar la materia de donde se obtiene la información
El alumno no demuestra haber obtenido los datos necesarios en los patrones o necesidades que precisan de focalización. La recopilación de información sobre la situación de salud concreta es pobre y desestructurada. Los diagnósticos son imprecisos y no se rodean de todas las evidencias necesarias

El documento describe adecuada, pero no suficientemente, la situación de salud (indicadores sociodemográficos y sanitarios) de la población de la zona. Además, apenas existe análisis e interpretación de los datos
El alumno demuestra haber obtenido los datos necesarios en algunos de los patrones o necesidades que precisan de focalización. La recopilación de información sobre la situación de salud concreta es correcta, pero insuficiente. Los diagnósticos enunciados son correctos, pero no completos en su formulación o faltan diagnósticos

El documento describe adecuada y suficientemente la situación de salud (indicadores sociodemográficos y sanitarios) de la población de la zona. Existe análisis e interpretación de los datos, pero es escasa o con bajo respaldo científico
El alumno demuestra haber obtenido los datos necesarios en todos los patrones o necesidades que precisan de focalización. La recopilación de información sobre la situación de salud concreta es correcta y suficiente. Los diagnósticos enunciados son correctos, pero no completos en su formulación o faltan diagnósticos

\section{El documento describe} adecuada y suficientemente la situación de salud (indicadores sociodemográficos y sanitarios) de la población de la zona. Existe análisis e interpretación de los datos con suficiente respaldo científico, pero se queda sobre todo en lo comparativo
El alumno demuestra haber obtenido los datos necesarios en todos los patrones o necesidades que precisan de focalización. La recopilación de información sobre la situación de salud concreta es correcta y suficiente. Los diagnósticos enunciados son correctos, completos en su formulación y saturan la situación de salud del paciente/usuario

El documento describe
adecuada y suficientemente
la situación de salud
(indicadores socio-
demográficos y sanitarios)
de la población de la zona.
Existe análisis de los datos
con buen respaldo científico,
y la interpretación es alta,
proporcionando explicaciones
posibles de lo observado

do el portafolio final (en modalidad de carpeta). Por otra parte, se llevaron a cabo al menos dos contactos por periodo con los tutores clínicos. Las profesoras también tuvieron tres reuniones a lo largo del desarrollo del proyecto, y contactos semanales vía correo electrónico.

En el curso académico 2006-2007 fue pilotado el portafolio con un estudiante de segundo curso y dos de tercero, quienes realizaron sus prácticas clínicas de enfermería comunitaria en el último periodo. Dicho pilotaje permitió precisar el diseño definitivo, así como las cuestiones logísticas para su puesta en marcha.

Las variables estudiadas fueron: edad y sexo del alumno, curso, ámbito (área sanitaria y centro de salud) y turno (mañana/tarde) en el que 
Tabla II. Características de la población estudiada $(n=38)$.

\begin{tabular}{clcc}
\hline Edad (media \pm DE) & \multicolumn{2}{c}{$21,5 \pm 4,39$ años } \\
\hline \multirow{4}{*}{ Sexo } & Mujer & $n$ & $\%$ \\
& Hombre & 7 & 81,6 \\
& & & 18,4 \\
\hline \multirow{4}{*}{ Edad } & 18-20 años & 24 & 63,2 \\
& 21-23 años & 8 & 21,0 \\
& 31-44 años & 5 & 13,2 \\
& & 1 & 2,6 \\
Curso & Segundo & 19 & 50,0 \\
& Tercero & 19 & 50,0 \\
\hline \multirow{2}{*}{ Turno } & Mañana & 27 & 71,0 \\
& Tarde & 11 & 29,0 \\
\hline
\end{tabular}

llevaban a cabo sus prácticas clínicas; puntualidad en las entregas de trabajos (en tiempo, fuera de plazo 1 hora, entre 1 y 6 horas, entre 6 y 24 horas y más de 24 horas); dificultad percibida en el realización del portafolio (de 1, ninguna hasta, 5, máxima); utilidad percibida de la realización del portafolio (de 1 , ninguna, hasta 5 , máxima); mejora del aprendizaje percibida (sí/no); horas dedicadas/semana del estudiante; calificaciones intermedias y finales en los trabajos que componían el portafolio (de 0 a 10); autoevaluación de las prácticas clínicas (de 0 a 10); calificación de la tutora de prácticas clínicas (de 0 a 10), y evaluación final del portafolio (de 0 a 10).

Para la calificación final de las prácticas clínicas se creó un índice sintético que incluía todas las calificaciones finales ponderadas (portafolio, $70 \%$, e informe de evaluación de la tutora clínica, $30 \%)$. Dentro del portafolio, el diario reflexivo aportaba un $30 \%$, el caso clínico un $20 \%$, el ASC un $20 \%$, y la autoevaluación del estudiante un $30 \%$. El peso otorgado a cada calificación se decidió mediante consenso del equipo de profesoras y tomando en consideración las experiencias de la Universitat de Manresa y de la Universitat Pompeu Fabra.

Respecto al tratamiento y análisis de los datos, se calcularon índices de estadística descriptiva en el análisis univariante. Para el análisis bivariante se hizo uso de pruebas no paramétricas, tanto para datos independientes (coeficiente de correlación de Spearman, $U$ de Mann-Whitney, test de Kruskal-Wallis) como para datos apareados (test de rangos de Wilcoxon, prueba de Friedman). Se calcularon intervalos de confianza para un 95\% (IC 95\%) de seguridad. Se utilizó el programa SPSS v. 15.0 para llevar a cabo el tratamiento estadístico.

\section{Resultados}

Participaron en el estudio 38 estudiantes, con una media de edad de $21,5 \pm 4,3$ años, y el $63,2 \%$ $(n=24)$ se encontraba entre los 18 y 20 años. El $81,6 \%$ eran mujeres $(n=31)$. Realizaron sus prácticas en turno de mañana el $71 \%(n=27)$ y procedía un $50 \%(n=19)$ de cada curso (Tabla II).

Respecto a las entregas intermedias de trabajos incluidos en el portafolio, fueron puntuales el $92 \%(n=35)$, el $78,9 \%(n=30)$ y el $65,8 \%(n=$ 25 ) en su primera, segunda y tercera entregas de trabajos, respectivamente $(p=0,005)$. Si bien este descenso en la puntualidad también se observó tanto para segundo como para el tercer curso, sólo fue significativo en segundo $(p=0,021)$. Para el $64,8 \%(n=24)$ la dificultad fue alta ( $>3$ en la escala de 1 a 5). La utilidad fue señalada como alta ( $>3$ en la escala de 1 a 5$)$ en el $73 \%(n=27)$. Mejoró el aprendizaje para el $100 \%(n=35$; tres personas no contestaron) de los estudiantes. La media de horas dedicadas a la semana a la elaboración del portafolio fue de $8,1 \pm 4,0 \mathrm{~h}$ (IC $95 \%=$ 6,6-9,5 h). La media de la evaluación de la tutora fue $8,1 \pm 1,1$ (IC 95\% = 7,7-8,5); la autoevaluación de los estudiantes fue de 7,6 $\pm 0,7$ (IC 95\% = $7,4-8,0)$, y la calificación definitiva del portafolio final por parte de las profesoras, de 7,2 $\pm 0,8$ (IC $95 \%=6,9-7,4)$. Las calificaciones de las tutoras fueron estadísticamente más altas para los estudiantes de tercero: segundo curso, 7,7 (IC 95\% $=7,0-8,3)$; tercer curso, 8,6 (IC 95\% = 8,2-9,0); $p=0,003$. No había diferencias estadísticamente significativas entre los cursos para ninguna de las restantes variables exploradas (Tabla III).

En el análisis longitudinal se pudo observar un aumento significativo $(p=0,000)$ de la calificación del diario reflexivo a lo largo de las entregas. En el caso clínico y el ASC se produjo un 
Tabla III. Indicadores del portafolio según curso.

\begin{tabular}{|c|c|c|c|c|}
\hline & Segundo curso & Tercer curso & Total & $p^{\text {a }}$ \\
\hline \multicolumn{5}{|l|}{ Puntualidad en la primera entrega de trabajos $(n, \%)^{\text {b }}$} \\
\hline En tiempo & $19(100)$ & $16(84,2)$ & $35(92,1)$ & \multirow{2}{*}{0,230} \\
\hline Fuera de plazo & 0 & $3(15,8)$ & $3(7,8)$ & \\
\hline \multicolumn{5}{|l|}{ Puntualidad en la segunda entrega de trabajos $(n, \%)^{\text {b }}$} \\
\hline En tiempo & $16(84,2)$ & $14(73,7)$ & $30(78,9)$ & \multirow{2}{*}{0,693} \\
\hline Fuera de plazo & $3(15,8)$ & $5(26,3)$ & $8(21,1)$ & \\
\hline \multicolumn{5}{|l|}{ Puntualidad en la tercera entrega de trabajos $(n, \%)^{\mathrm{b}}$} \\
\hline En tiempo & $13(68,4)$ & $12(63,2)$ & $25(65,8)$ & \multirow{2}{*}{1,000} \\
\hline Fuera de plazo & $6(31,6)$ & $7(36,8)$ & $13(34,2)$ & \\
\hline \multicolumn{5}{|l|}{ Dificultad percibida en la realización del portafolio $(n, \%)^{c}$} \\
\hline Hasta 3 & $6(31,6)$ & $7(38,9)$ & $13(35,2)$ & \multirow[b]{2}{*}{0,737} \\
\hline Más de 3 & $13(68,4)$ & $11(61,1)$ & $24(64,8)$ & \\
\hline \multicolumn{5}{|l|}{ Utilidad percibida de la realización del portafolio $(n, \%)^{c}$} \\
\hline Hasta 3 & $7(36,8)$ & $3(16,7)$ & $10(27,0)$ & \multirow{2}{*}{0,269} \\
\hline Más de 3 & $12(63,2)$ & $15(83,3)$ & $27(73,0)$ & \\
\hline \multicolumn{5}{|l|}{ Mejora de aprendizaje percibida con la realización del portafolio $(n, \%)$} \\
\hline Sí & $18(100)$ & $17(100)$ & $35(92,0)$ & \multirow{2}{*}{ NA } \\
\hline No & 0 & 0 & $3(8,0)$ & \\
\hline Tiempo (h/semana) dedicado al portafolio (media \pm DE; IC 95\%) & $8,4 \pm 4,9(5,7-11,2)$ & $7,6 \pm 3,3(5,6-9,6)$ & $8,1 \pm 4,0(6,6-9,5)$ & 0,832 \\
\hline Calificación final del diario reflexivo (media $\pm \mathrm{DE} ; \mathrm{IC} 95 \%)^{\mathrm{d}}$ & $7,1 \pm 1,0(6,6-7,7)$ & $7,2 \pm 0,9(6,6-7,7)$ & $7,2 \pm 0,9(6,9-7,6)$ & 0,425 \\
\hline Calificación final del caso clínico (media \pm DE; IC 95\%) ${ }^{d}$ & $6,7 \pm 1,1(6,0-7,3)$ & $6,3 \pm 1,0(5,7-6,9)$ & $6,3 \pm 1,6(5,7-6,8)$ & 0,105 \\
\hline $\begin{array}{l}\text { Calificación final del análisis de salud de la comunidad } \\
\text { (media } \pm \text { DE; IC 95\%) }^{d}\end{array}$ & $6,3 \pm 1,3(5,6-7,0)$ & $6,3 \pm 0,8(5,8-6,7)$ & $6,3 \pm 1,0(6,0-6,7)$ & 0,525 \\
\hline Calificación del tutor clínico (media \pm DE; IC 95\%) d & $7,7 \pm 1,1(7,0-8,3)$ & $8,6 \pm 0,7(8,2-9,0)$ & $8,1 \pm 1,1(7,7-8,5)$ & 0,003 \\
\hline Calificación de la autoevaluación (media \pm DE; IC 95\%) d & $7,6 \pm 0,6(7,2-8,0)$ & $7,6 \pm 0,7(7,2-8,0)$ & $7,6 \pm 0,7(7,4-8,0)$ & 0,196 \\
\hline Calificación final del portafolio (media \pm DE; IC 95\%) ${ }^{d}$ & $7,0 \pm 0,7(6,6-7,5)$ & $7,4 \pm 0,6(7,1-7,8)$ & $7,2 \pm 0,8(6,9-7,4)$ & 0,053 \\
\hline
\end{tabular}

${ }^{a}$ Comparación entre segundo y tercer curso; ${ }^{b}$ Valor $p$ de la comparación entre las tres entregas: segundo curso $(0,021)$, tercer curso $(0,180)$, total $(0,005) ;{ }^{~}$ En una escala de 1 (ninguna) hasta 5 (máxima); ¿ Para la calificación se utilizó una escala de 0 (mínima) a 10 (máxima). DE: desviación estándar; IC: intervalo de confianza; NA: no aplicable.

descenso significativo $(p=0,000)$ entre la primera entrega y la final (Tabla IV).

Existía una correlación significativa entre la evaluación final del portafolio y la autoevaluación de los estudiante ( $r h o=0,624 ; p=0,000$ ), la evaluación final del portafolio y la calificación de la tutora (rho $=0,505 ; p=0,002$ ) y la autoevaluación del estudiante y la calificación de la tutora (rho = $0,488 ; p=0,004)$. La edad se correlacionaba positivamente con las tres calificaciones (Tabla V).

No se mostraron diferencias estadísticamente significativas entre hombres y mujeres en la calificación del tutor, en la autoevaluación ni en la calificación del portafolio final. Tampoco existían diferencias en estas calificaciones atendiendo al turno (mañana/tarde) en el que se realizaron las prácticas clínicas.

\section{Discusión}

La implementación del portafolio ha puesto de manifiesto la dificultad sentida por los estudiantes en un proceso que requiere escritura reflexiva, trabajo diario y autoevaluación. Es de destacar el 
Tabla IV. Calificaciones de los trabajos que componían el portafolio según entregas (media \pm desviación estándar).

\begin{tabular}{lccccc}
\hline & 1. $^{\text {a entrega }}$ & 2. ${ }^{\text {e entrega }}$ & 3. ${ }^{\text {a entrega }}$ & Entrega final & $p$ \\
\hline Diario reflexivo $(n=36)$ & $5,8 \pm 1,2$ & $6,0 \pm 1,0$ & $6,4 \pm 1,0$ & $7,1 \pm 0,9$ & 0,000 \\
\hline Caso clínico $(n=33)^{\text {a }}$ & $6,8 \pm 1,4$ & $5,7 \pm 0,7$ & & $6,2 \pm 1,5$ & 0,000 \\
\hline Análisis de salud de la comunidad $^{b}$ & & $7,0 \pm 1,2$ & & $6,3 \pm 1,1$ & 0,000 \\
\hline
\end{tabular}

a Sólo hubo dos entregas intermedias; ${ }^{b}$ Sólo hubo una entrega intermedia.

Tabla V. Correlación entre las calificaciones del portafolio, de la tutora y del estudiante, y de cada una de ellas con la edad ${ }^{\text {. }}$.

\begin{tabular}{lccc}
\hline & Portafolio final & Autoevaluación & Tutora \\
\hline Autoevaluación & 0,624 & & \\
\hline Tutora & 0,505 & 0,488 & \\
\hline Edad & 0,580 & 0,485 & 0,590 \\
\hline
\end{tabular}

aTodos los coeficientes de correlación de la tabla (rho) tuvieron un valor $p<0,05$

paralelismo entre 'dificultad' y 'utilidad', de manera que ambos indicadores se incrementan de forma similar, pero lo verdaderamente reseñable es que todos los estudiantes reflejaron que el portafolio mejoraba su aprendizaje. La dificultad se traduce en una alta percepción de utilidad y de mejora para el aprendizaje. Estos resultados son consistentes con los señalados en otros trabajos [10-12], así como en una reciente revisión sistemática [16] que pone de manifiesto que el uso de portafolio mejora el conocimiento y la comprensión e incrementa la autoconciencia, la participación y la reflexión. Pero también se refleja en la bibliografía que el tiempo que se precisa para la elaboración del portafolio puede ser una dificultad para otros aprendizajes clínicos [17].

El hecho de que el número de borradores se fuera incrementando a medida que transcurría el periodo de prácticas puede explicar la disminución de la puntualidad a lo largo de las entregas.

Las calificaciones del diario reflexivo fueron mejorando significativamente a lo largo de las entregas. El hecho de no evidenciar esta tendencia en las calificaciones del caso clínico y el ASC puede relacionarse con la insuficiente monitorización y consiguiente proceso de devolución por parte de las profesoras en estos trabajos. Se pidieron menos entregas que en el diario reflexivo para no sobrecargar al estudiante. Fundamentalmente se les exigía que entregaran un borrador o una presentación de lo que iban a ser esos trabajos. En dichos borradores no se podían evaluar gran parte de los indicadores propuestos y, por lo tanto, la media obtenida estaba prácticamente impregnada de los indicadores A (ortografía), $\mathrm{B}$ (sintaxis) y C (uso de lenguaje adecuado). Es obvio que esto supone un sesgo en la medición $y$, por lo tanto, resta validez a las calificaciones intermedias de estos dos trabajos.

Entre ambos cursos sólo se objetivaron diferencias estadísticamente significativas en lo que se refiere a la evaluación del tutor, siendo aquellos que tutorizaban en tercer curso quienes otorgaban calificaciones más altas. Los autores apuntan algunas interpretaciones que serían susceptibles de indagarse con mayor profundidad en futuros estudios. Por una parte, los estudiantes de segundo año cuentan con un menor conocimiento clínico y un peor desenvolvimiento técnico a la hora de realizar sus prácticas clínicas, pero es probable que el tutor tenga las mismas expectativas que con el estudiante de tercer año. Esto 
podría responder a un desconocimiento de los objetivos y competencias específicas para cada curso por parte de los profesionales que realizan la tutorización. Por otro lado, en el último año de la carrera, el estudiante puede llegar a desarrollar con su tutor una relación cercana a la de colegas, el alumno lleva a cabo acciones con más pericia, tiene mayor capacidad para tomar decisiones; está, en otras palabras, casi a la altura de un profesional. Esta realidad puede condicionar más la evaluación y favorecer la tendencia a la sobrestimación.

Si bien las calificaciones de todos los actores (profesoras, tutoras y estudiantes) que intervienen en el proceso evaluativo del portafolio mostraron una aceptable correlación, es entre el estudiante y la profesora donde esta correlación es más fuerte. Un reciente estudio refleja que la concordancia entre estudiante y profesor es del 60\% [18].

El portafolio, como técnica pedagógica de construcción de conocimientos que incorpora una dinámica de reflexión y autoevaluación, integra parámetros de evaluación del aprendizaje y del desarrollo competencial, lo cual es una demanda tanto del ámbito académico como del ámbito clínico, con la finalidad última de contar con enfermeros y enfermeras competentes para brindar cuidados a la comunidad.

\section{Bibliografía}

1. Ley Orgánica 4/2007, de 12 de abril, por la que se modifica la Ley Orgánica 6/2001 de Universidades. Boletín Oficial del Estado, n. ${ }^{\circ}$ 89, de 13 de abril de 2007.

2. Real Decreto $1393 / 2007$, de 29 de octubre, por el que se establece la ordenación de las enseñanzas universitarias oficiales. Boletín Oficial del Estado, n. ${ }^{\circ} 260$, de 30 de octubre de 2007.

3. De la Cruz-Tomé MA. El proceso de convergencia europeo: ocasión de modernizar la universidad española si se produce un cambio de mentalidad en gestores, profesores y estudiantes. Aula Abierta 2003; 82: 191-216.

4. Barragán-Sánchez R. El portafolio, metodología de evaluación y aprendizaje de cara al nuevo EEES. Una experiencia práctica en la Universidad de Sevilla. Revista Latinoamericana de Tecnología Educativa 2005; 4: 121-39.

5. Artel JA, Spandel V. Using portfolios of students work in instruction and assessment. Portland, OR: Northwest Regional Education Laboratory; 1991.
6. Villarini A. Primer seminario taller sobre fundamentos y principios de evaluación auténtica. República Dominicana: Facultad Autónoma de Santo Domingo; 1996.

7. Vizcarro C. Algunas aportaciones para la evaluación mediante portafolios. Documentación aportada en el Diploma en Innovación y Nuevas Tecnologías en Educación Superior. Curso 2001/02. Madrid: Centro de Aprendizaje e Instrucción. Universidad Autónoma de Madrid; 2002.

8. Beckley WL. Creating a classroom portfolio system. A guide to assist classroom teachers in kindergarten through eighth grade. Dubuque, Iowa: Kendall/Hunt Publishing; 1997.

9. Olivé-Ferrer MC. Implementación del portafolio del estudiante en la asignatura 'Fonaments d'infermeria' como instrumento para el aprendizaje reflexivo y crítico. Educare21 2004; 13. URL: http://enfermeria21.com/ educare/educare13/ensenando/ensenando4.htm.

10. Vera-Cortés M, Canalejas-Pérez C. El portafolio como recurso de aprendizaje e instrumento de evaluación de estudiantes repetidores de enfermería. Educ Med 2007; 10: 114-20.

11. López-Ortega J, López-Sánchez FJ, Sánchez-Jurado LI, Calero-García MJ. El uso del portafolio para la enseñanza en Enfermería. Experiencia de tres cursos académicos. Ini Inv 2008 (revista electrónica de la Universidad de Jaén). URL: http://virtual.ujaen.es/ininv/images/ ficheros/art_investigacion/num_e3_1008/e3a26.pdf.

12. Canalejas-Pérez C, Cid-Galán ML, Martínez-Martín ML, Martín-Salinas C. El portafolios como instrumento de aprendizaje y evaluación de competencias en los estudiantes de enfermería. Enferm Clin 2009; 19: 16-23.

13. Farr R, Tone B. Portfolio and performance assessment: helping students evaluate their progress as readers and writers. 2 ed. Orlando, FL: Harcourt Brace; 1998.

14. Bordas I, Cabrera FA. Estrategias de evaluación de los aprendizajes centrados en el proceso. Revista Española de Pedagogía 2001; 218: 25-48.

15. Castelló-Badía M, Monereo-Font C. L’avaluació per carpetes en el pràcticum de psicopedagogia. Barcelona: Universitat Autònoma de Barcelona; 2000.

16. Buckley S, Coleman J, Davison I, Khan KS, Zamora J, Malick S, et al. The educational effects of portfolios on undergraduate student learning: a Best Evidence Medical Education (BEME) systematic review. BEME Guide No. 11. Med Teach 2009; 31: 282-98.

17. Davis MH, Ponnamperuma GG, Ker JS. Student perceptions of a portfolio assessment process. Med Educ 2009; 43: 89-98.

18. Chamorro-Rebollo E, Sendino-Revuelta A, San José-Valiente B. Concordancia entre alumnos y profesores en las puntuaciones de exámenes teóricos. Metas Enferm 2007; 10: 25-30. 